\title{
Tolerant or intolerant character of interacting criteria in aggregation by the Choquet integral ${ }^{*}$
}

\author{
Jean-Luc Marichal \\ Department of Mathematics, Brigham Young University \\ 292 TMCB, Provo, Utah 84602, U.S.A. \\ marichal [at] math. byu. edu \\ Submitted 26 February 2002, Revised 3 October 2002
}

\begin{abstract}
In many multi-criteria decision-making problems the decision criteria present some interaction whose nature may vary from one situation to another. For example, some criteria may be statistically correlated, thus making them somewhat redundant or opposed. Some others may be somewhat substitutive or complementary depending on the behavior of the decision maker. Some others may be decisive in the sense that the global score (of any alternative) obtained by aggregation is bounded by the partial score along one of them.

In this paper we analyze this latter form of interaction. When a criterion bounds the global score from above, it is called a blocker or a veto, due to its rather intolerant character. When it bounds the global score from below, it is then called a pusher or a favor. We thus investigate the tolerance of criteria, or equivalently, the tolerance of the weighted aggregation operator (here the Choquet integral) that is used to aggregate criteria. More specifically, we propose (axiomatically) indices to appraise the extent to which each criterion behaves like a veto or a favor in the aggregation by the Choquet integral. Previous to this, we also propose global tolerance degrees measuring the extent to which the Choquet integral is conjunctive or disjunctive.
\end{abstract}

Keywords: multi-criteria analysis; interacting criteria; fuzzy measure; Choquet integral.

\section{Introduction}

The use of the fuzzy measures in multi-criteria decision-making enables us to model some interaction phenomena existing among criteria; see [10, 20]. For example, when two criteria are positively correlated then the importance of these criteria, taken together, should be strictly less than the sum of the importances of the single criteria.

Of course, there are interaction phenomena whose nature is not linked to correlation. In [20] the author discusses the concepts of substitutiveness and complementarity. Two

\footnotetext{
*This paper is a revised and extended version with proofs of some parts of the book chapter [19].
} 
criteria are said to be substitutive if the satisfaction of only one of them produces the same effect as the satisfaction of both. In that case the importance of the two criteria taken together is equal to the importance of each of them. By contrast, two criteria are said to be complementary if the satisfaction of only one of them does not produce any effect. In that case the importance of each criterion alone is null. Contrary to correlation phenomena, these latter interaction forms cannot be detected by observing a score table. They just represent the opinion of the decision maker on the relative importance of criteria, independently of the partial scores obtained by the alternatives along these criteria.

Another form of dependence among criteria corresponds to the presence of veto or favor criteria [10]. A criterion is a veto (resp. a favor) if the partial score of any alternative along this criterion bounds from above (resp. from below) the global score obtained by aggregation.

For example, consider the problem of evaluating students with respect to various courses (criteria) and suppose that there exists a veto (resp. favor) course. This means that the global grade obtained by any student cannot be greater than (resp. less than) the grade obtained at this course.

We thus see that, in some respect, veto criteria are intolerant and favor criteria are tolerant. Even though such an extreme behavior rarely occurs in practical applications, some criteria may have a certain degree of veto or favor.

In this paper we analyze the tolerance degree of criteria, locally and globally. That is, we propose (axiomatically) veto and favor indices giving the extent to which a given criterion behaves like a veto or a favor. We also propose global indices, called andness and orness degrees, that measure the overall tolerance of criteria. These latter indices also give the degree to which the aggregation is conjunctive or disjunctive.

We assume here that criteria are all expressed on the same interval scale, and hence we aggregate them by means of the discrete Choquet integral, which has been proved to be the best suitable operator to aggregate interacting criteria defined on such a scale type (see Theorem 2.1 below). Thus the main aim of this paper is to investigate the average tolerance degree of criteria as well as the tolerance degree of each of them when they are aggregated by the Choquet integral.

The outline of this paper is as follows. In $\S 2$ we recall the definition of the Choquet integral and its use in multi-criteria decision aid. We also present some of its particular cases, namely the weighted arithmetic mean, the ordered weighted averaging, and the partial minimum and maximum. In $\S 3$ we define global tolerance degrees of criteria as andness and orness degrees of the Choquet integral. $\S 4$ is then devoted to veto and favor degrees which are characterized by means of rather natural properties. Finally, in $\S 5$ we demonstrate the use of all these indices on a practical example.

Throughout this paper, the label set $N=\{1, \ldots, n\}$ represents the set of criteria of a given decision problem. To avoid a heavy notation we will often omit braces for singletons, e.g., writing $a(i), N \backslash i$ instead of $a(\{i\}), N \backslash\{i\}$. Moreover, cardinality of subsets $S, T, \ldots$ will be denoted whenever possible by the corresponding lower case letters $s, t, \ldots$, otherwise by the standard notation $|S|,|T|, \ldots$.

For any subset $S \subseteq N, \mathbf{1}_{S}$ will denote the characteristic vector of $S$ in $\{0,1\}^{n}$, i.e., the vector of $\{0,1\}^{n}$ whose $i$ th component is 1 if and only if $i \in S$. 


\section{The Choquet integral and its particular cases}

The use of the Choquet integral has been proposed by many authors as an adequate substitute to the weighted arithmetic mean to aggregate interacting criteria; see e.g. [9, 20]. In the weighted arithmetic mean model, each criterion $i \in N$ is given a weight $\omega_{i} \in[0,1]$ representing the importance of this criterion in the decision. In the Choquet integral model, where criteria can be dependent, a fuzzy measure [28] is used to define a weight on each combination of criteria, thus making it possible to model the interaction existing among criteria.

Definition 2.1 A fuzzy measure on $N$ is a set function $v: 2^{N} \rightarrow[0,1]$, that is nondecreasing with respect to set inclusion and such that $v(\varnothing)=0$ and $v(N)=1$.

For any $S \subseteq N, v(S)$ can be regarded as the weight of importance of the combination $S$ of criteria. This interpretation will be illustrated in the remark following Theorem 2.1.

Throughout, we will denote by $\mathcal{F}_{N}$ the set of all fuzzy measures on $N$, and by $\Pi_{N}$ the set of permutations on $N$.

For any fuzzy measure $v \in \mathcal{F}_{N}$, the dual fuzzy measure $v^{*} \in \mathcal{F}_{N}$ is defined by $v^{*}(S)=$ $1-v(N \backslash S)$; see $[10,13]$. Obviously, we have $\left(v^{*}\right)^{*}=v$ for any $v \in \mathcal{F}_{N}$.

For any fuzzy measure $v \in \mathcal{F}_{N}$ and any permutation $\pi \in \Pi_{N}, \pi v$ will denote the fuzzy measure of $\mathcal{F}_{N}$ defined by $\pi v(\pi(S))=v(S)$ for all $S \subseteq N$, where $\pi(S)=\{\pi(i) \mid i \in S\}$.

We now give the definition of the Choquet integral. Although this concept was first introduced in capacity theory [3], its use as a (fuzzy) integral with respect to a fuzzy measure was proposed by Höhle [15] and rediscovered later by Murofushi and Sugeno [24, 25].

Definition 2.2 Let $v \in \mathcal{F}_{N}$. The (discrete) Choquet integral of $x: N \rightarrow \mathbb{R}$ with respect to $v$ is defined by

$$
\mathcal{C}_{v}(x):=\sum_{i=1}^{n} x_{(i)}\left[v\left(A_{(i)}\right)-v\left(A_{(i+1)}\right)\right]
$$

where $(\cdot)$ indicates a permutation on $N$ such that $x_{(1)} \leq \ldots \leq x_{(n)}$. Also $A_{(i)}=\{(i), \ldots,(n)\}$, and $A_{(n+1)}=\varnothing$.

Thus defined, the Choquet integral has very good properties for aggregation (see e.g. Grabisch [9]). For instance, it is continuous, non decreasing, comprised between min and max, stable under the same transformations of interval scales in the sense of the theory of measurement, and coincides with the weighted arithmetic mean (discrete Lebesgue integral) as soon as the fuzzy measure is additive. Moreover, in $[18,20]$ the author proposed an axiomatic characterization of the class of all the Choquet integrals with $n$ arguments. The statement is the following:

Theorem 2.1 The operators $M_{v}: \mathbb{R}^{n} \rightarrow \mathbb{R}\left(v \in \mathcal{F}_{N}\right)$ are

- linear w.r.t. the fuzzy measures, that is, there exist $2^{n}$ functions $f_{T}: \mathbb{R}^{n} \rightarrow \mathbb{R}(T \subseteq N)$, such that

$$
M_{v}=\sum_{T \subseteq N} v(T) f_{T} \quad\left(v \in \mathcal{F}_{N}\right)
$$

- non decreasing in each argument, 
- stable for the admissible positive linear transformations, that is,

$$
M_{v}\left(r x+s \mathbf{1}_{N}\right)=r M_{v}(x)+s
$$

for all $x \in \mathbb{R}^{n}, r>0, s \in \mathbb{R}$,

- properly weighted by $v$, that is,

$$
M_{v}\left(\mathbf{1}_{S}\right)=v(S) \quad(S \subseteq N),
$$

if and only if $M_{v}=\mathcal{C}_{v}$ for all $v \in \mathcal{F}_{N}$.

The axioms presented in the previous characterization are natural enough in the context of multi-criteria decision-making. The first one is proposed to keep the aggregation model as simple as possible. The second axiom says that increasing a partial score along any criterion cannot decrease the global score. The third axiom only demands that the aggregated value is stable with respect to any change of scale, provided that the partial scores are defined on an interval scale. Finally, assuming that the partial score scale is embedded in $[0,1]$, the fourth axiom suggests that the weight of importance of any subset $S$ of criteria is defined as the global evaluation of the alternative that completely satisfies criteria $S$ and totally fails to satisfy the others. This latter axiom is fundamental. It actually gives an appropriate definition of the weights of subsets of criteria, interpreting them as global evaluations of particular profiles.

The following proposition $[14, \S 4]$ (see also $[16, \S 4]$ ) gives the link between the Choquet integral $\mathcal{C}_{v}$ defined from a given fuzzy measure $v \in \mathcal{F}_{N}$ and the Choquet integral $\mathcal{C}_{v^{*}}$ defined from the dual measure $v^{*}$.

Proposition 2.1 For any $v \in \mathcal{F}_{N}$, we have

$$
1-\mathcal{C}_{v}\left(1-x_{1}, \ldots, 1-x_{n}\right)=\mathcal{C}_{v^{*}}\left(x_{1}, \ldots, x_{n}\right) \quad\left(x \in \mathbb{R}^{n}\right) .
$$

Now, the Möbius transform of a given fuzzy measure $v \in \mathcal{F}_{N}$ is a set function $a: 2^{N} \rightarrow \mathbb{R}$ defined by

$$
a(S)=\sum_{T \subseteq S}(-1)^{s-t} v(T) \quad(S \subseteq N) .
$$

The transformation is invertible and we have (see e.g. [26])

$$
v(S)=\sum_{T \subseteq S} a(T) \quad(S \subseteq N) .
$$

The Möbius transform enables us to express some functions of $v$ in a simpler form. For example, the Choquet integral is written $[2,21]$

$$
\mathcal{C}_{v}(x)=\sum_{T \subseteq N} a(T) \bigwedge_{i \in T} x_{i} \quad\left(x \in \mathbb{R}^{n}\right) .
$$

Rewriting Eq. (4) for $v^{*}$ and then using Eq. (1), we also have

$$
\mathcal{C}_{v}(x)=\sum_{T \subseteq N} a^{*}(T) \bigvee_{i \in T} x_{i} \quad\left(x \in \mathbb{R}^{n}\right),
$$


where $a^{*}$ is the Möbius transform of the dual fuzzy measure $v^{*}$.

Note that the conversion relationship between $a$ and $a^{*}$ is given by the following two formulas:

$$
\begin{aligned}
a^{*}(T) & =(-1)^{t+1} \sum_{K \supseteq T} a(K) & (\varnothing \neq T \subseteq N), \\
a(T) & =(-1)^{t+1} \sum_{K \supseteq T} a^{*}(K) & (\varnothing \neq T \subseteq N) ;
\end{aligned}
$$

see [18, Prop. 4.2.3] (see also [12, §6]).

We now present some subclasses of Choquet integrals. Any vector $\omega \in[0,1]^{n}$ such that $\sum_{i} \omega_{i}=1$ will be called a weight vector as we go on.

\subsection{The weighted arithmetic mean}

Definition 2.3 For any weight vector $\omega \in[0,1]^{n}$, the weighted arithmetic mean operator $\mathrm{WAM}_{\omega}$ associated to $\omega$ is defined by

$$
\operatorname{WAM}_{\omega}(x)=\sum_{i=1}^{n} \omega_{i} x_{i} .
$$

We can easily see that $\mathrm{WAM}_{\omega}$ is a Choquet integral $\mathcal{C}_{v}$ with respect to an additive fuzzy measure:

$$
v(S)=\sum_{i \in S} \omega_{i} \quad(S \subseteq N) .
$$

The corresponding Möbius representation is given by

$$
\left\{\begin{array}{l}
a(i)=\omega_{i}, \quad \forall i \in N \\
a(S)=0, \quad \forall S \subseteq N \text { such that } s \geq 2 .
\end{array}\right.
$$

Conversely, the weights associated to $\mathrm{WAM}_{\omega}$ are given by

$$
\omega_{i}=v(i)=a(i) \quad(i \in N) .
$$

\subsection{The ordered weighted averaging operator}

Yager [29] defined in 1988 the ordered weighted averaging operators (OWA) as follows.

Definition 2.4 For any weight vector $\omega \in[0,1]^{n}$, the ordered weighted averaging operator $\mathrm{OWA}_{\omega}$ associated to $\omega$ is defined by

$$
\operatorname{OWA}_{\omega}(x)=\sum_{i=1}^{n} \omega_{i} x_{(i)}
$$

with convention that $x_{(1)} \leq \cdots \leq x_{(n)}$.

The following result, due to Grabisch [8] (see [21] for a shorter proof), shows that any OWA operator is a Choquet integral w.r.t. a fuzzy measure that depends only on the cardinality of subsets. 
Proposition 2.2 Let $v \in \mathcal{F}_{N}$. Then the following assertions are equivalent:

i) For any $S, S^{\prime} \subseteq N$ such that $|S|=\left|S^{\prime}\right|$, we have $v(S)=v\left(S^{\prime}\right)$.

ii) There exists a weight vector $\omega$ such that $\mathcal{C}_{v}=\mathrm{OWA}_{\omega}$.

iii) $\mathcal{C}_{v}$ is a symmetric function.

The fuzzy measure $v$ associated to $\mathrm{OWA}_{\omega}$ is given by

$$
v(S)=\sum_{i=n-s+1}^{n} \omega_{i} \quad(S \subseteq N, S \neq \varnothing),
$$

and its Möbius representation by [11, Thm 1]

$$
a(S)=\sum_{j=0}^{s-1}\left(\begin{array}{c}
s-1 \\
j
\end{array}\right)(-1)^{s-1-j} \omega_{n-j} \quad(S \subseteq N, \quad S \neq \varnothing) .
$$

Conversely, the weights associated to $\mathrm{OWA}_{\omega}$ are given by

$$
\omega_{n-s}=v(S \cup i)-v(S)=\sum_{T \subseteq S} a(T \cup i) \quad(i \in N, S \subseteq N \backslash i) .
$$

The class of ordered weighted averaging operators $\mathrm{OWA}_{\omega}$ includes an important subclass, namely the order statistics

$$
\mathrm{OS}_{k}(x)=x_{(k)},
$$

when $\omega_{k}=1$ for some $k \in N$. In this case, we have, for any $S \subseteq N$,

$$
\begin{aligned}
& v(S)= \begin{cases}1, & \text { if } s \geq n-k+1, \\
0, & \text { otherwise, }\end{cases} \\
& a(S)= \begin{cases}(-1)^{s-n+k-1}\left(\begin{array}{l}
s-1 \\
n-k
\end{array}\right), & \text { if } s \geq n-k+1, \\
0, & \text { otherwise }\end{cases}
\end{aligned}
$$

Since, for any $k \in N$, we have

$$
1-\mathrm{OS}_{k}\left(1-x_{1}, \ldots, 1-x_{n}\right)=\mathrm{OS}_{n-k+1}\left(x_{1}, \ldots, x_{n}\right) \quad\left(x \in \mathbb{R}^{n}\right),
$$

from (1) it follows that, for any $S \subseteq N$,

$$
\begin{aligned}
& v^{*}(S)= \begin{cases}1, & \text { if } s \geq k, \\
0, & \text { otherwise, }\end{cases} \\
& a^{*}(S)= \begin{cases}(-1)^{s-k}\left(\begin{array}{l}
s-1 \\
k-1
\end{array}\right), & \text { if } s \geq k, \\
0, & \text { otherwise. }\end{cases}
\end{aligned}
$$

\subsection{The partial minimum and maximum operators}

Definition 2.5 For any non-empty subset $T \subseteq N$, the partial minimum operator $\min _{T}$ and the partial maximum operator $\max _{T}$, associated to $T$, are respectively defined by

$$
\begin{aligned}
\min _{T}(x) & =\min _{i \in T} x_{i}, \\
\max _{T}(x) & =\max _{i \in T} x_{i} .
\end{aligned}
$$


For the operator $\min _{T}$, we have, for any $S \subseteq N$,

$$
\begin{aligned}
& v(S)= \begin{cases}1, & \text { if } S \supseteq T, \\
0, & \text { else },\end{cases} \\
& a(S)= \begin{cases}1, & \text { if } S=T, \\
0, & \text { else. }\end{cases}
\end{aligned}
$$

To refer easily to these particular set functions, we will henceforth denote them by $\underline{v}_{T}$ and $\underline{a}_{T}$, respectively.

For the operator $\max _{T}$, we have, for any $S \subseteq N$,

$$
\begin{aligned}
& v(S)= \begin{cases}1, & \text { if } S \cap T \neq \varnothing, \\
0, & \text { else, }\end{cases} \\
& a(S)= \begin{cases}(-1)^{s+1}, & \text { if } \varnothing \neq S \subseteq T, \\
0, & \text { else. }\end{cases}
\end{aligned}
$$

We will denote these latter set functions by $\bar{v}_{T}$ and $\bar{a}_{T}$, respectively.

We can readily see that each of the fuzzy measures $\underline{v}_{T}$ and $\bar{v}_{T}$ is the dual of the other, that is, $\bar{v}_{T}=\underline{v}_{T}^{*}$ and $\underline{v}_{T}=\bar{v}_{T}^{*}$ for any $T \neq \varnothing$. Thus, the expression of $\bar{a}_{T}(S)$ follows immediately from Eq. (5).

We also have the following interesting result:

Proposition 2.3 For any $T \subseteq N, T \neq \varnothing$, we have

$$
\begin{aligned}
\bar{v}_{T} & =\sum_{\substack{K \subseteq T \\
K \neq \varnothing}}(-1)^{k+1} \underline{v}_{K} \\
\underline{v}_{T} & =\sum_{\substack{K \subseteq T \\
K \neq \varnothing}}(-1)^{k+1} \bar{v}_{K}
\end{aligned}
$$

Proof. We have, for any $S \subseteq N$,

$$
\begin{aligned}
\sum_{\substack{K \subseteq T \\
K \neq \varnothing}}(-1)^{k+1} \underline{v}_{K}(S) & =\sum_{\substack{K \subseteq S \cap T \\
K \neq \varnothing}}(-1)^{k+1} \\
& = \begin{cases}0, & \text { if } S \cap T=\varnothing \\
\sum_{i=1}^{|S \cap T|}\left(\begin{array}{c}
|S \cap T| \\
k
\end{array}\right)(-1)^{k+1}=1, & \text { otherwise }\end{cases}
\end{aligned}
$$

which proves (11). On the other hand, by (11), we have, for any $S \subseteq N$,

$$
\begin{aligned}
\sum_{\substack{K \subseteq T \\
K \neq \varnothing}}(-1)^{k+1} \bar{v}_{K}(S) & =\sum_{\substack{K \subseteq T \\
K \neq \varnothing}}(-1)^{k+1} \sum_{\substack{J \subseteq K \\
J \neq \varnothing}}(-1)^{j+1} \underline{v}_{J}(S) \\
& =\sum_{\substack{J \subseteq T \\
J \neq \varnothing}} \underline{v}_{J}(S) \sum_{K: J \subseteq K \subseteq T}(-1)^{k-j} \\
& =\underline{v}_{T}(S),
\end{aligned}
$$

which proves (12). 
Note that if $\psi(v)$ is a function that is linear with respect to $v$ then, from Eqs. (11) and (12) we immediately have

$$
\begin{aligned}
& \psi\left(\bar{v}_{T}\right)=\sum_{\substack{K \subseteq T \\
K \neq \varnothing}}(-1)^{k+1} \psi\left(\underline{v}_{K}\right) \\
& \psi\left(\underline{v}_{T}\right)=\sum_{\substack{K \subseteq T \\
K \neq \varnothing}}(-1)^{k+1} \psi\left(\bar{v}_{K}\right)
\end{aligned}
$$

For example, if $\psi(v)=\mathcal{C}_{v}$ then we have

$$
\begin{aligned}
\max _{T}(x) & =\sum_{\substack{K \subseteq T \\
K \neq \varnothing}}(-1)^{k+1} \min _{K}(x) \quad\left(x \in \mathbb{R}^{n}\right) \\
\min _{T}(x) & =\sum_{\substack{K \subseteq T \\
K \neq \varnothing}}(-1)^{k+1} \max _{K}(x) \quad\left(x \in \mathbb{R}^{n}\right)
\end{aligned}
$$

\section{Conjunction and disjunction degrees}

Consider the cube $[0,1]^{n}$ as a probability space with uniform distribution. Then the expected value of $\mathcal{C}_{v}(x)$, that is

$$
\mathrm{E}\left(\mathcal{C}_{v}\right):=\int_{[0,1]^{n}} \mathcal{C}_{v}(x) \mathrm{d} x
$$

represents the average value of the Choquet integral $\mathcal{C}_{v}$ over $[0,1]^{n}$. This expression gives the average position of $\mathcal{C}_{v}$ within the interval $[0,1]$.

For example, from the identity

$$
\int_{[0,1]^{n}{ }_{i \in S}} \bigwedge_{i} x_{i} \mathrm{~d} x=\frac{1}{s+1} \quad(S \subseteq N)
$$

(see $[13, \S 4])$ and the obvious property

$$
\mathrm{E}\left(\mathcal{C}_{v^{*}}\right)=1-\mathrm{E}\left(\mathcal{C}_{v}\right)
$$

(see Eq. (1)) we obtain

$$
\mathrm{E}(\min )=\frac{1}{n+1} \quad \text { and } \quad \mathrm{E}(\max )=\frac{n}{n+1}
$$

Now, since the Choquet integral is always internal to the set of its arguments, that is

$$
\min x_{i} \leq \mathcal{C}_{v}(x) \leq \max x_{i} \quad\left(x \in[0,1]^{n}\right),
$$

from (13) it follows that

$$
\mathrm{E}(\min ) \leq \mathrm{E}\left(\mathcal{C}_{v}\right) \leq \mathrm{E}(\max ) \quad\left(v \in \mathcal{F}_{N}\right) .
$$

Based on these observations, the author defined in [18] (see also [19]) the conjunction degree or the degree of andness of $\mathcal{C}_{v}$ as the relative position of $\mathrm{E}\left(\mathcal{C}_{v}\right)$ with respect to the lower bound of the interval $[\mathrm{E}(\min ), \mathrm{E}(\max )]$. 
Definition 3.1 The degree of andness of $\mathcal{C}_{v}$ is defined by

$$
\operatorname{andness}\left(\mathcal{C}_{v}\right):=\frac{\mathrm{E}(\max )-\mathrm{E}\left(\mathcal{C}_{v}\right)}{\mathrm{E}(\max )-\mathrm{E}(\min )}
$$

This value represents the degree to which the average value of $\mathcal{C}_{v}$ is close to that of "min". In some sense, it also reflects the extent to which $\mathcal{C}_{v}$ behaves like a minimum or has a conjunctive behavior.

Similarly, the relative position of $\mathrm{E}\left(\mathcal{C}_{v}\right)$ with respect to $\mathrm{E}(\max )$ is called the disjunction degree or the degree of orness of $\mathcal{C}_{v}$. It measures the degree to which $\mathcal{C}_{v}$ behaves like a maximum or has a disjunctive behavior.

Definition 3.2 The degree of orness of $\mathcal{C}_{v}$, is defined by

$$
\operatorname{orness}\left(\mathcal{C}_{v}\right):=\frac{\mathrm{E}\left(\mathcal{C}_{v}\right)-\mathrm{E}(\min )}{\mathrm{E}(\max )-\mathrm{E}(\min )}
$$

It should be noted that, historically, these two concepts have been introduced as early as 1974 by Dujmović $[5,6]$ in the particular case of the root-mean-power. Here we have simply applied his definitions to the Choquet integral ${ }^{1}$. Of course, these definitions can be applied to any internal function which is integrable in $[0,1]^{n}$. Note also that similar approaches have been proposed for some non-internal functions such as triangular norms and conorms; see [17].

By definition, both andness $\left(\mathcal{C}_{v}\right)$ and orness $\left(\mathcal{C}_{v}\right)$ lie in the unit interval $[0,1]$. Furthermore they fulfill the following properties:

$$
\begin{gathered}
\operatorname{andness}\left(\mathcal{C}_{v}\right)+\operatorname{orness}\left(\mathcal{C}_{v}\right)=1 \\
\operatorname{andness}\left(\mathcal{C}_{v^{*}}\right)=\operatorname{orness}\left(\mathcal{C}_{v}\right) \\
\operatorname{orness}\left(\mathcal{C}_{v^{*}}\right)=\operatorname{andness}\left(\mathcal{C}_{v}\right)
\end{gathered}
$$

The degree of orness is actually a measure of global tolerance of criteria or, equivalently, a measure of the tolerance of the decision maker. In fact, tolerant decision makers can accept that only some criteria are satisfied. This corresponds to a disjunctive behavior (orness $\left(\mathcal{C}_{v}\right)>0.5$ ), whose extreme example is max. On the other hand, intolerant decision makers demand that most criteria are satisfied. This corresponds to a conjunctive behavior (orness $\left.\left(\mathcal{C}_{v}\right)<0.5\right)$, whose extreme example is min. When orness $\left(\mathcal{C}_{v}\right)=0.5$ the decision maker is medium (neither tolerant nor intolerant).

Using Eqs. (4), (14), and (15), we easily obtain the following result:

Proposition 3.1 For any $v \in \mathcal{F}_{N}$, we have

$$
\begin{aligned}
\operatorname{orness}\left(\mathcal{C}_{v}\right) & =\frac{1}{n-1} \sum_{T \subseteq N} \frac{n-t}{t+1} a(T) \\
\operatorname{andness}\left(\mathcal{C}_{v}\right) & =\frac{1}{n-1} \sum_{T \subseteq N} \frac{n t-1}{t+1} a(T)
\end{aligned}
$$

where $a$ is the Möbius representation of $v$.

\footnotetext{
${ }^{1}$ An alternative definition of these concepts was proposed very recently [7]. It consists in normalizing the Choquet integral before calculating its expectation; that is,

$$
\operatorname{andness}^{\prime}\left(\mathcal{C}_{v}\right)=E\left[\frac{\max (x)-\mathcal{C}_{v}(x)}{\max (x)-\min (x)}\right] \quad \text { and } \quad \operatorname{orness}^{\prime}\left(\mathcal{C}_{v}\right)=E\left[\frac{\mathcal{C}_{v}(x)-\min (x)}{\max (x)-\min (x)}\right]
$$
}


In terms of the fuzzy measure $v$ the degree of orness takes the following form.

Proposition 3.2 For any $v \in \mathcal{F}_{N}$, we have

$$
\operatorname{orness}\left(\mathcal{C}_{v}\right)=\frac{1}{n-1} \sum_{T \varsubsetneqq N} \frac{(n-t) ! t !}{n !} v(T),
$$

or equivalently,

$$
\operatorname{orness}\left(\mathcal{C}_{v}\right)=\frac{1}{n-1} \sum_{t=1}^{n-1} \frac{1}{\left(\begin{array}{c}
n \\
t
\end{array}\right)} \sum_{\substack{T \subseteq N \\
|T|=t}} v(T)
$$

Proof. The result follows from the conversion formulas between the Shapley interaction index $I_{\mathrm{Sh}}(\varnothing)$ and $v$ (see Table 3 in [13]) and the fact that

$$
\mathrm{E}\left(\mathcal{C}_{v}\right)=\sum_{T \subseteq N} \frac{1}{t+1} a(T)
$$

where $a$ is the Möbius representation of $v$.

Corollary 3.1 Let $v \in \mathcal{F}_{N}$. Then

$$
\begin{aligned}
& \operatorname{orness}\left(\mathcal{C}_{v}\right)=0 \quad \Leftrightarrow \quad \mathcal{C}_{v}=\min \\
& \operatorname{orness}\left(\mathcal{C}_{v}\right)=1 \quad \Leftrightarrow \quad \mathcal{C}_{v}=\max
\end{aligned}
$$

Proof. If $\mathcal{C}_{v}=\min$ then orness $\left(\mathcal{C}_{v}\right)=0$ trivially. Conversely, if orness $\left(\mathcal{C}_{v}\right)=0$ then, by (20), we have $v(T)=0$ for all $T \subset N$, with $T \neq \varnothing$ and $T \neq N$, which proves the first equivalence.

By (1) and (15)-(17) we merely have

$$
\begin{aligned}
\operatorname{orness}\left(\mathcal{C}_{v}\right)=1 & \Leftrightarrow \operatorname{orness}\left(\mathcal{C}_{v^{*}}\right)=0 \\
& \Leftrightarrow \mathcal{C}_{v^{*}}=\min \\
& \Leftrightarrow \mathcal{C}_{v}=\max
\end{aligned}
$$

which proves the second equivalence.

The concept of orness was defined independently by Yager [29] in the particular case of OWA operators; see also [1,30]. His definition is based on the use of the so-called regular increasing monotone quantifiers, that is, increasing functions $Q:[0,1] \rightarrow[0,1]$, with $Q(0)=0$ and $Q(1)=1$, which represent linguistic quantifiers such as all, most, many, at least $k$. For any $k \in N, Q(k / n)$ indicates the lowest global evaluation of an alternative that fully satisfies $k$ criteria, that is,

$$
Q(k / n):=\operatorname{OWA}_{\omega}\left(\mathbf{1}_{\{1, \ldots, k\}}\right)=\sum_{i=1}^{k} \omega_{n-i+1} .
$$

Yager then defined

$$
\operatorname{orness}\left(\mathrm{OWA}_{\omega}\right):=\frac{1}{n-1} \sum_{k=1}^{n-1} Q(k / n)=\frac{1}{n-1} \sum_{k=1}^{n}(k-1) \omega_{k} .
$$




\begin{tabular}{|c|c|c|}
\hline $\mathcal{C}_{v}$ & $\operatorname{andness}\left(\mathcal{C}_{v}\right)$ & $\operatorname{orness}\left(\mathcal{C}_{v}\right)$ \\
\hline $\mathrm{WAM}_{\omega}$ & $1 / 2$ & $1 / 2$ \\
$\mathrm{OWA}_{\omega}$ & $\frac{1}{n-1} \sum_{i=1}^{n}(n-i) \omega_{i}$ & $\frac{1}{n-1} \sum_{i=1}^{n}(i-1) \omega_{i}$ \\
$\mathrm{OS}_{k}$ & $\frac{n-k}{n-1}$ & $\frac{k-1}{n-1}$ \\
$\min _{T}$ & $\frac{n t-1}{(n-1)(t+1)}$ & $\frac{n-t}{(n-1)(t+1)}$ \\
$\max _{T}$ & $\frac{n-t}{(n-1)(t+1)}$ & $\frac{n t-1}{(n-1)(t+1)}$ \\
\hline
\end{tabular}

Table 1: Degrees of andness and orness for various Choquet integrals

These definitions can be adapted to the more general case of the Choquet integrals. The average value of the lowest global evaluation over all the alternatives that fully satisfy $k$ criteria is given by

$$
Q(k / n):=\frac{1}{\left(\begin{array}{l}
n \\
k
\end{array}\right)} \sum_{\substack{K \subseteq N \\
|K|=k}} \mathcal{C}_{v}\left(\mathbf{1}_{K}\right) \quad(k \in N) .
$$

The orness of $\mathcal{C}_{v}$ can then be defined by

$$
\operatorname{orness}\left(\mathcal{C}_{v}\right):=\frac{1}{n-1} \sum_{k=1}^{n-1} Q(k / n),
$$

and we retrieve $(20)$.

Table 1 gives the degrees of andness and orness for particular Choquet integrals.

It could also be interesting to appraise the dispersion of $\mathcal{C}_{v}$ around its typical value by calculating its variance, that is,

$$
\sigma^{2}\left(\mathcal{C}_{v}\right):=\int_{[0,1]^{n}}\left[\mathcal{C}_{v}(x)-\mathrm{E}\left(\mathcal{C}_{v}\right)\right]^{2} \mathrm{~d} x
$$

or equivalently,

$$
\sigma^{2}\left(\mathcal{C}_{v}\right)=\mathrm{E}\left(\mathcal{C}_{v}^{2}\right)-\mathrm{E}\left(\mathcal{C}_{v}\right)^{2}
$$

From this definition it follows that

$$
\sigma^{2}\left(\mathcal{C}_{v^{*}}\right)=\sigma^{2}\left(\mathcal{C}_{v}\right)
$$
of $v$.

The following result gives the expression of $\sigma^{2}\left(\mathcal{C}_{v}\right)$ in terms of the Möbius representation 
Proposition 3.3 For any $v \in \mathcal{F}_{N}$, we have

$$
\sigma^{2}\left(\mathcal{C}_{v}\right)=\sum_{S, T \subseteq N} a(S) a(T) \frac{s+t-|S \cup T|}{(s+1)(t+1)(|S \cup T|+2)} .
$$

Proof. It has been proved in $[18, \S 7.2]$ that, for any $S, T \subseteq N$, there holds

$$
\int_{[0,1]^{n}}\left(\bigwedge_{i \in S} x_{i}\right)\left(\bigwedge_{j \in T} x_{j}\right) \mathrm{d} x=\frac{s+t+2}{(s+1)(t+1)(|S \cup T|+2)}
$$

We then have

$$
\begin{aligned}
\mathrm{E}\left(\mathcal{C}_{v}^{2}\right) & =\sum_{S, T \subseteq N} a(S) a(T) \int_{[0,1]^{n}}\left(\bigwedge_{i \in S} x_{i}\right)\left(\bigwedge_{j \in T} x_{j}\right) \mathrm{d} x \\
& =\sum_{S, T \subseteq N} a(S) a(T) \frac{s+t+2}{(s+1)(t+1)(|S \cup T|+2)}
\end{aligned}
$$

and

$$
\mathrm{E}\left(\mathcal{C}_{v}\right)^{2}=\sum_{S, T \subseteq N} a(S) a(T) \frac{1}{(s+1)(t+1)} .
$$

Eq. (21) then allows us to conclude.

For example, we have

$$
\begin{aligned}
\sigma^{2}\left(\mathrm{WAM}_{\omega}\right) & =\frac{1}{12} \sum_{i=1}^{n} \omega_{i}^{2} \\
\sigma^{2}\left(\min _{T}\right) & =\frac{t}{(t+1)^{2}(t+2)} \\
\sigma^{2}\left(\max _{T}\right) & =\frac{t}{(t+1)^{2}(t+2)} .
\end{aligned}
$$

\section{$4 \quad$ Veto and favor effects}

Interesting behavioral phenomena in aggregation of criteria are the veto and favor effects. Both were introduced recently in multi-criteria decision-making by Grabisch [10] ${ }^{2}$.

A criterion $k \in N$ is said to be a veto or a blocker for $\mathcal{C}_{v}$ if

$$
\mathcal{C}_{v}(x) \leq x_{k} \quad\left(x \in[0,1]^{n}\right)
$$

This definition is motivated by the fact that the non satisfaction of a veto criterion should entail a low global score.

Similarly, the criterion $k$ is a favor or a pusher for $\mathcal{C}_{v}$ if

$$
\mathcal{C}_{v}(x) \geq x_{k} \quad\left(x \in[0,1]^{n}\right) .
$$

In this case, the satisfaction of criterion $k$ entails necessarily a high global score.

\footnotetext{
${ }^{2}$ The concepts of veto and favor actually predate their use by Grabisch. Historically, these concepts were already proposed in 1991 by Dubois and Koning [4] in the context of social choice functions, where "favor" was called "dictator".
} 
It is easy to see that $k$ is a veto (resp. favor) for $\mathcal{C}_{v}$ if and only if it is a favor (resp. veto) for $\mathcal{C}_{v^{*}}$.

A consequence of these definitions is that no Choquet integral can model simultaneously a veto on a criterion and a favor on another one. Indeed we clearly have

$$
x_{i} \leq \mathcal{C}_{v}(x) \leq x_{j} \forall x \in[0,1]^{n} \Rightarrow i=j .
$$

We can also see that if the same criterion $k$ is simultaneously a veto and a favor, then it is a dictator. Indeed, we then have

$$
\mathcal{C}_{v}(x)=x_{k} \quad\left(x \in[0,1]^{n}\right) .
$$

Note that if the decision maker considers that a given criterion must absolutely be satisfied (veto criterion), then (s)he is conjunctive oriented. Indeed, by integrating both sides of Eq. $(22)$ we clearly have orness $\left(\mathcal{C}_{v}\right) \leq 0.5$. Similarly, if the decision maker considers that the satisfaction of a given criterion is sufficient (favor criteria) then (s)he is disjunctive oriented. From Eq. (23) we have orness $\left(\mathcal{C}_{v}\right) \geq 0.5$.

The following result provides equivalent conditions for $k$ to be a veto for $\mathcal{C}_{v}$. The third one is due to Grabisch [10].

Proposition 4.1 Let $k \in N$ and $v \in \mathcal{F}_{N}$. Then the following eight assertions are equivalent:

i) $k$ is a veto for $\mathcal{C}_{v}$

ii) $\mathcal{C}_{v}(x) \leq x_{k} \forall x \in\{0,1\}^{n}$

iii) $v(T)=0$ whenever $T \not \supset k$

iv) $v(N \backslash k)=0$

v) $\forall \lambda \in[0,1], \forall x \in[0,1]^{n}, x_{k} \leq \lambda \Rightarrow \mathcal{C}_{v}(x) \leq \lambda$

vi) $\forall \lambda \in[0,1], \mathcal{C}_{v}\left(\mathbf{1}_{N \backslash k}+\lambda \mathbf{1}_{k}\right)=\lambda$

vii) $\exists \lambda \in[0,1)$ such that $\forall x \in[0,1]^{n}$ we have $x_{k} \leq \lambda \Rightarrow \mathcal{C}_{v}(x) \leq \lambda$

viii) $\exists \lambda \in[0,1)$ such that $\mathcal{C}_{v}\left(\mathbf{1}_{N \backslash k}+\lambda \mathbf{1}_{k}\right)=\lambda$

Proof. We shall prove the equivalence by establishing the chain of implications:

$$
i) \Rightarrow i i) \Rightarrow i i i) \Rightarrow i v) \Rightarrow v i) \Rightarrow i) \Rightarrow v) \Rightarrow v i i) \Rightarrow v i i i) \Rightarrow i v)
$$

which seems to provide the shortest proof.

i) $\Rightarrow$ ii) Trivial.

$i i) \Rightarrow$ iii) If $T \not \supset k$ then $v(T)=\mathcal{C}_{v}\left(\mathbf{1}_{T}\right) \leq\left(\mathbf{1}_{T}\right)_{k}=0$.

iii) $\Rightarrow i v)$ Trivial.

$i v) \Rightarrow v i)$ By the stability property of $\mathcal{C}_{v}$, we have

$$
\mathcal{C}_{v}\left(\mathbf{1}_{N \backslash k}+\lambda \mathbf{1}_{k}\right)=\lambda+(1-\lambda) \mathcal{C}_{v}\left(\mathbf{1}_{N \backslash k}\right)=\lambda+(1-\lambda) v(N \backslash k)=\lambda .
$$

$v i) \Rightarrow i$ ) By increasing monotonicity of $\mathcal{C}_{v}$, we have

$$
\mathcal{C}_{v}(x) \leq \mathcal{C}_{v}\left(\mathbf{1}_{N \backslash k}+x_{k} \mathbf{1}_{k}\right)=x_{k}
$$

for all $x \in[0,1]^{n}$.

i) $\Rightarrow v$ ) We merely have $\mathcal{C}_{v}(x) \leq x_{k} \leq \lambda$.

$v) \Rightarrow v i i) \Rightarrow v i i i)$ Trivial. 
viii) $\Rightarrow$ iv) We have

$$
\lambda=\mathcal{C}_{v}\left(\mathbf{1}_{N \backslash k}+\lambda \mathbf{1}_{k}\right)=\lambda+(1-\lambda) \mathcal{C}_{v}\left(\mathbf{1}_{N \backslash k}\right)
$$

and hence $0=\mathcal{C}_{v}\left(\mathbf{1}_{N \backslash k}\right)=v(N \backslash k)$.

The equivalence between $i$ ) and vii) in Proposition 4.1 is surprising. By imposing that $\mathcal{C}_{v}(x) \leq \lambda$ whenever $x_{k} \leq \lambda$ for a given threshold $\lambda \in[0,1)$, we necessarily consider $k$ as a veto for $\mathcal{C}_{v}$. For instance, consider again the problem of evaluating students with respect to different subjects (courses) and suppose that the teacher of course $k$ decides that if a student gets a mark less than 18/20 for course $k$ then the global mark over all courses must be less than 18/20. In this case, this teacher has a veto behavior with respect to his/her colleagues.

More surprising is the following phenomenon. Suppose that a student gets $x=11 / 20$ for course $k$ and $y=12 / 20$ everywhere else and that it is decided that the global mark is $x=11 / 20$. Then $k$ is a veto. Indeed, we simply have

$$
\begin{aligned}
x & =\mathcal{C}_{v}\left(y \mathbf{1}_{N \backslash k}+x \mathbf{1}_{k}\right)=x+(y-x) \mathcal{C}_{v}\left(\mathbf{1}_{N \backslash k}\right) \\
& =x+(y-x) v(N \backslash k),
\end{aligned}
$$

retrieving condition $i v$ ). Thus, increasing the marks on courses $N \backslash k$ has no effect on the global evaluation. This clearly indicates a form of interaction among courses.

Proposition 4.1 can be easily rewritten for favor criteria as follows:

Proposition 4.2 Let $k \in N$ and $v \in \mathcal{F}_{N}$. Then the following eight assertions are equivalent:

i) $k$ is a favor for $\mathcal{C}_{v}$

ii) $\mathcal{C}_{v}(x) \geq x_{k} \forall x \in\{0,1\}^{n}$

iii) $v(T)=1$ whenever $T \ni k$

iv) $v(k)=1$

v) $\forall \lambda \in[0,1], \forall x \in[0,1]^{n}, x_{k} \geq \lambda \Rightarrow \mathcal{C}_{v}(x) \geq \lambda$

vi) $\forall \lambda \in[0,1], \mathcal{C}_{v}\left(\lambda \mathbf{1}_{k}\right)=\lambda$

vii) $\exists \lambda \in(0,1]$ such that $\forall x \in[0,1]^{n}$ we have $x_{k} \geq \lambda \Rightarrow \mathcal{C}_{v}(x) \geq \lambda$

viii) $\exists \lambda \in(0,1]$ such that $\mathcal{C}_{v}\left(\lambda \mathbf{1}_{k}\right)=\lambda$

Now, it seems sensible to define indices that measure the degree of veto or favor of a given criterion $j \in N$. A natural definition of a degree of veto (resp. favor) consists in considering the probability

$$
\operatorname{Pr}\left[\mathcal{C}_{v}(x) \leq x_{j}\right] \quad\left(\text { resp. } \operatorname{Pr}\left[\mathcal{C}_{v}(x) \geq x_{j}\right]\right)
$$

where $x \in[0,1]^{n}$ is a multi-dimensional random variable uniformly distributed. Unfortunately, such an index does not depend always continuously on $v$. For example, one can easily show that (see Appendix A)

$$
\operatorname{Pr}\left[\operatorname{WAM}_{\omega}(x) \leq x_{j}\right]= \begin{cases}1, & \text { if } \omega_{j}=1 \\ 1 / 2, & \text { otherwise }\end{cases}
$$

Thinking of condition $i$ ) above we could as well consider the same probability but with $x \in\{0,1\}^{n}$. However this leads to the same drawback (see Appendix A). 
An alternative approach consists in defining such indices axiomatically. In [18] the author introduced the following indices, based on an axiomatic characterization:

$$
\begin{gathered}
\operatorname{veto}\left(\mathcal{C}_{v}, j\right):=1-\frac{1}{n-1} \sum_{T \subseteq N \backslash j} \frac{(n-t-1) ! t !}{(n-1) !} v(T) \\
\operatorname{favor}\left(\mathcal{C}_{v}, j\right):=\frac{1}{n-1} \sum_{T \subseteq N \backslash j} \frac{(n-t-1) ! t !}{(n-1) !} v(T \cup j)-\frac{1}{n-1}
\end{gathered}
$$

In terms of the Möbius representation of $v$, these indices are written:

$$
\begin{gathered}
\operatorname{veto}\left(\mathcal{C}_{v}, j\right)=1-\frac{n}{n-1} \sum_{T \subseteq N \backslash j} \frac{1}{t+1} a(T) \\
\operatorname{favor}\left(\mathcal{C}_{v}, j\right)=\frac{n}{n-1} \sum_{T \subseteq N \backslash j} \frac{1}{t+1}[a(T \cup j)+a(T)]-\frac{1}{n-1}
\end{gathered}
$$

Moreover, we can easily prove that

$$
\begin{aligned}
& \operatorname{veto}\left(\mathcal{C}_{v^{*}}, j\right)=\operatorname{favor}\left(\mathcal{C}_{v}, j\right) \\
& \text { favor }\left(\mathcal{C}_{v^{*}}, j\right)=\operatorname{veto}\left(\mathcal{C}_{v}, j\right)
\end{aligned}
$$

Although the form of these indices seems not very informative, the axiomatic that supports them is rather natural. We present it in the following theorem. The proof is given in Appendix B.

Theorem 4.1 The numbers $\psi\left(\mathcal{C}_{v}, j\right)\left(j \in N, v \in \mathcal{F}_{N}\right)$

- are linear w.r.t. the fuzzy measures, that is, there exist real constants $p_{T}^{j}(T \subseteq N)$ such that

$$
\psi\left(\mathcal{C}_{v}, j\right)=\sum_{T \subseteq N} p_{T}^{j} v(T) \quad\left(j \in N, v \in \mathcal{F}_{N}\right)
$$

- fulfill the "symmetry" axiom, that is, for any permutation $\pi \in \Pi_{N}$, we have

$$
\psi\left(\mathcal{C}_{v}, j\right)=\psi\left(\mathcal{C}_{\pi v}, \pi(j)\right) \quad\left(j \in N, v \in \mathcal{F}_{N}\right)
$$

- fulfill the "boundary" axiom, that is, for any $T \subseteq N, T \neq \varnothing$, and any $j \in T$, we have

$$
\psi\left(\min _{T}, j\right)=1, \quad\left(\operatorname{resp} . \psi\left(\max _{T}, j\right)=1\right)
$$

- fulfill the "normalization" axiom, that is, for any $v \in \mathcal{F}_{N}$,

$$
\begin{gathered}
\psi\left(\mathcal{C}_{v}, i\right)=\psi\left(\mathcal{C}_{v}, j\right) \quad \forall i, j \in N \\
\Downarrow \\
\psi\left(\mathcal{C}_{v}, j\right)=\operatorname{andness}\left(\mathcal{C}_{v}\right) \quad\left(\text { resp. orness }\left(\mathcal{C}_{v}\right)\right) \quad \forall j \in N
\end{gathered}
$$

if and only if $\psi\left(\mathcal{C}_{v}, j\right)=\operatorname{veto}\left(\mathcal{C}_{v}, j\right)\left(\right.$ resp. favor $\left.\left(\mathcal{C}_{v}, j\right)\right)$ for all $j \in N$ and all $v \in \mathcal{F}_{N}$. 
Let us comment on the axioms presented in this characterization. As for Choquet integral, we ask the veto and favor indices to be linear with respect to the fuzzy measures. We also require that these indices be independent of the numbering of criteria. Next, the boundary axiom is motivated by the observation that any $j \in T$ is a veto (resp. favor) criterion for $\min _{T}\left(\right.$ resp. $\left.\max _{T}\right)$. Finally, the normalization axiom says that if the degree of veto (resp. favor) does not depend on criteria, then it identifies with the degree of intolerance (resp. tolerance) of the decision maker.

Thus defined, we see that veto $\left(\mathcal{C}_{v}, j\right)$ is more or less the degree to which the decision maker demands that criterion $j$ is satisfied. Similarly, favor $\left(\mathcal{C}_{v}, j\right)$ is the degree to which the decision maker considers that a good score along criterion $j$ is sufficient to be satisfied.

It is easy to observe that $\operatorname{veto}\left(\mathcal{C}_{v}, j\right) \in[0,1]$ and favor $\left(\mathcal{C}_{v}, j\right) \in[0,1]$. Furthermore, we have, for any $v \in \mathcal{F}_{N}$,

$$
\begin{aligned}
\frac{1}{n} \sum_{j=1}^{n} \operatorname{veto}\left(\mathcal{C}_{v}, j\right) & =\operatorname{andness}\left(\mathcal{C}_{v}\right), \\
\frac{1}{n} \sum_{j=1}^{n} \operatorname{favor}\left(\mathcal{C}_{v}, j\right) & =\operatorname{orness}\left(\mathcal{C}_{v}\right) .
\end{aligned}
$$

We also have

$$
\operatorname{veto}\left(\mathcal{C}_{v}, j\right)+\operatorname{favor}\left(\mathcal{C}_{v}, j\right)=1+\frac{n \phi(v, j)-1}{n-1} \quad\left(j \in N, v \in \mathcal{F}_{N}\right),
$$

where $\phi(v, j)$ is the Shapley importance index of criterion $j$ with respect to $v[27]$ (see also $[20])$, that is

$$
\phi(v, i):=\sum_{T \subseteq N \backslash i} \frac{(n-t-1) ! t !}{n !}[v(T \cup i)-v(T)] .
$$

Eq. (24) shows that if both $\operatorname{veto}\left(\mathcal{C}_{v}, j\right)$ and favor $\left(\mathcal{C}_{v}, j\right)$ increase then so does the importance $\phi(v, j)$.

Table 2 gives the veto and favor indices for particular Choquet integrals.

\section{An illustrative example}

In this final section we give an example, borrowed from Grabisch [9]. Let us consider the problem of evaluating students in a high school with respect to three subjects: mathematics $(\mathrm{M})$, physics $(\mathrm{P})$, and literature $(\mathrm{L})$. Usually, this is done by a simple weighted arithmetic mean, whose weights are the coefficients of importance of the different subjects. Suppose that the school is more scientifically than literary oriented, so that weights could be for example proportional to 3,3 , and 2 , respectively. Then the weighted arithmetic mean will give the following results for three students $a, b$, and $c$ (marks are given on a scale from 0 to 20$)$ :

\begin{tabular}{|c|ccc|c|}
\hline Student & M & P & L & Global \\
\hline$a$ & 18 & 16 & 10 & 15.25 \\
$b$ & 10 & 12 & 18 & 12.75 \\
$c$ & 14 & 15 & 15 & 14.625 \\
\hline
\end{tabular}




\begin{tabular}{|c|c|c|}
\hline $\mathcal{C}_{v}$ & $\operatorname{veto}\left(\mathcal{C}_{v}, j\right)$ & favor $\left(\mathcal{C}_{v}, j\right)$ \\
\hline $\mathrm{WAM}_{\omega}$ & $\frac{1}{2}+\frac{n\left(\omega_{j}-1 / n\right)}{2(n-1)}$ & $\frac{1}{2}+\frac{n\left(\omega_{j}-1 / n\right)}{2(n-1)}$ \\
\hline $\mathrm{OWA}_{\omega}$ & $\frac{1}{n-1} \sum_{i=1}^{n}(n-i) \omega_{i}$ & $\frac{1}{n-1} \sum_{i=1}^{n}(i-1) \omega_{i}$ \\
\hline $\mathrm{OS}_{k}$ & $\frac{n-k}{n-1}$ & $\frac{k-1}{n-1}$ \\
\hline $\min _{T}$ & $\begin{cases}1, & \text { if } j \in T \\
\frac{n t-t-1}{(n-1)(t+1)}, & \text { otherwise, }\end{cases}$ & $\begin{array}{ll}\frac{n-t}{(n-1) t}, & \text { if } j \in T \\
\frac{n-t-1}{(n-1)(t+1)}, & \text { otherwise, }\end{array}$ \\
\hline $\max _{T}$ & $\begin{cases}\frac{n-t}{(n-1) t}, & \text { if } j \in T \\
\frac{n-t-1}{(n-1)(t+1)}, & \text { otherwise, }\end{cases}$ & $\begin{array}{ll}1, & \text { if } j \in T \\
\frac{n t-t-1}{(n-1)(t+1)}, & \text { otherwise. }\end{array}$ \\
\hline
\end{tabular}

Table 2: Veto and favor indices for various Choquet integrals

If the school wants to favor well equilibrated students without weak points then student $c$ should be considered better than student $a$, who has a severe weakness in literature. Unfortunately, no weight vector $\left(\omega_{\mathrm{M}}, \omega_{\mathrm{P}}, \omega_{\mathrm{L}}\right)$ satisfying $\omega_{\mathrm{M}}=\omega_{\mathrm{P}}>\omega_{\mathrm{L}}$ is able to favor student $c$. Indeed, we have:

$$
c \succ a \Leftrightarrow \omega_{\mathrm{L}}>\omega_{\mathrm{M}} .
$$

The reason of this problem is that too much importance is given to mathematics and physics, which present some overlap effect since, usually, students good at mathematics are also good at physics (and vice versa), so that the evaluation is overestimated (resp. underestimated) for students good (resp. bad) at mathematics and/or physics. This problem can be solved by using a suitable fuzzy measure $v$ and the Choquet integral.

- Since scientific subjects are more important than literature, the following weights can be put on subjects taken individually: $v(\mathrm{M})=v(\mathrm{P})=0.45$ and $v(\mathrm{~L})=0.3$. Note that the initial ratio of weights $(3,3,2)$ is kept unchanged.

- Since mathematics and physics overlap, the weights attributed to the pair $\{\mathrm{M}, \mathrm{P}\}$ should be less than the sum of the weights of mathematics and physics: $v(\mathrm{MP})=0.5$.

- Since students equally good at scientific subjects and literature must be favored, the weight attributed to the pair $\{L, M\}$ should be greater than the sum of individual weights (the same for physics and literature): $v(\mathrm{ML})=v(\mathrm{PL})=0.9$. 
- $v(\varnothing)=0$ and $v(\mathrm{MPL})=1$ by definition.

The Möbius representation is then given by

$$
\begin{array}{llll}
a(\varnothing)=0 & a(\mathrm{M})=0.45 & a(\mathrm{MP})=-0.40 & a(\mathrm{MPL})=-0.10 \\
& a(\mathrm{P})=0.45 & a(\mathrm{ML})=0.15 \\
& a(\mathrm{~L})=0.30 & a(\mathrm{PL})=0.15
\end{array}
$$

Applying Choquet integral with the above fuzzy measure leads to the following new global evaluations:

\begin{tabular}{|c|ccc|c|}
\hline Student & $\mathrm{M}$ & $\mathrm{P}$ & $\mathrm{L}$ & Global \\
\hline$a$ & 18 & 16 & 10 & 13.9 \\
$b$ & 10 & 12 & 18 & 13.6 \\
$c$ & 14 & 15 & 15 & 14.6 \\
\hline
\end{tabular}

The expected result is then obtained. Also remark that student $b$ has still the lowest rank, as requested by the scientific tendency of this high school.

Now, let us turn to a deeper analysis of the orientation of the school or its director. From the fuzzy measure proposed, we obtain the following Shapley indices and degrees of veto and favor:

\begin{tabular}{|c|ccc|}
\cline { 2 - 4 } \multicolumn{1}{c|}{} & $\mathrm{M}$ & $\mathrm{P}$ & $\mathrm{L}$ \\
\hline $\operatorname{veto}\left(\mathcal{C}_{v}, i\right)$ & 0.362 & 0.362 & 0.525 \\
favor $\left(\mathcal{C}_{v}, i\right)$ & 0.575 & 0.575 & 0.600 \\
$\phi(v, i)$ & 0.292 & 0.292 & 0.417 \\
\hline $\operatorname{veto}\left(\mathcal{C}_{v}, i\right) / \operatorname{andness}\left(\mathcal{C}_{v}\right)$ & 0.868 & 0.868 & 1.259 \\
$\operatorname{favor}\left(\mathcal{C}_{v}, i\right) / \operatorname{orness}\left(\mathcal{C}_{v}\right)$ & 0.986 & 0.986 & 1.029 \\
$n * \phi(v, i)$ & 0.875 & 0.875 & 1.250 \\
\hline
\end{tabular}

Looking at the veto and favor degrees, we observe that the school seems to favor slightly the students (disjunctive oriented). This is in accordance with the degree of orness

$$
\operatorname{orness}\left(\mathcal{C}_{v}\right)=0.583
$$

As we can see, it is convenient to scale the veto degrees (resp. the favor degrees, the Shapley indices) by the factor andness $\left(\mathcal{C}_{v}\right)^{-1}$ (resp. the factor orness $\left(\mathcal{C}_{v}\right)^{-1}$, the factor $n$ ), so that a value greater than one indicates a veto degree (resp. a favor degree, a Shapley index) more important than the average.

We also have $\mathrm{E}\left(\mathcal{C}_{v}\right)=0.542$ and $\sigma\left(\mathcal{C}_{v}\right)=0.180$, showing that the global evaluation is rather dispersed around its typical value 0.542 .

Remark that all these behavioral parameters have been obtained from a given fuzzy measure. In many practical situations, the fuzzy measure is not completely available. We might then fix its values from information on behavioral parameters; see [22, 23]. 


\section{Concluding remarks}

In this paper we have mainly investigated the tolerance of interacting criteria when aggregated by the Choquet integral. More precisely, we have introduced global tolerance indices, called andness and orness, measuring the degree to which the Choquet integral has a conjunctive or disjunctive character. Then, we have proposed and axiomatized individual indices, called veto and favor, measuring the degree to which each criterion behaves like a blocker or a pusher. These indices make it possible to identify and measure the "dictatorial" tendency of criteria, which is a particular interaction phenomenon.

\section{A The veto index as a probability}

Proposition A.1 i) If $x \in[0,1]^{n}$ is a multi-dimensional random variable uniformly distributed, then

$$
\operatorname{Pr}\left[\operatorname{WAM}_{\omega}(x) \leq x_{j}\right]= \begin{cases}1, & \text { if } \omega_{j}=1 \\ 1 / 2, & \text { otherwise }\end{cases}
$$

ii) If $x \in\{0,1\}^{n}$ is a multi-dimensional random variable uniformly distributed, then

$$
\operatorname{Pr}\left[\operatorname{WAM}_{\omega}(x) \leq x_{j}\right]=\frac{1}{2}+\left(\frac{1}{2}\right)^{1+r},
$$

where $r=\left|\left\{i \in N \backslash j: \omega_{i} \neq 0\right\}\right|$.

Proof. $i$ ) The case $\omega_{j}=1$ is trivial. If $\omega_{j}<1$ then we have

$$
\begin{aligned}
& \operatorname{Pr}\left[\sum_{i=1}^{n} \omega_{i} x_{i} \leq x_{j}\right]=\operatorname{Pr}\left[\sum_{\substack{i=1 \\
i \neq j}}^{n} \omega_{i} x_{i} \leq x_{j}\left(1-\omega_{j}\right)\right] \\
= & \operatorname{Pr}\left[\frac{1}{1-\omega_{j}} \sum_{\substack{i=1 \\
i \neq j}}^{n} \omega_{i} x_{i} \leq x_{j}\right]=1-\operatorname{Pr}\left[\frac{1}{1-\omega_{j}} \sum_{\substack{i=1 \\
i \neq j}}^{n} \omega_{i} x_{i}>x_{j}\right] \\
= & 1-\int_{0}^{1} \cdots \int_{0}^{1} \frac{1}{1-\omega_{j}} \sum_{\substack{i=1 \\
i \neq j}}^{n} \omega_{i} x_{i} \mathrm{~d} x_{1} \cdots \mathrm{d} x_{j-1} \mathrm{~d} x_{j+1} \cdots \mathrm{d} x_{n} \\
= & 1-\frac{1}{2\left(1-\omega_{j}\right)} \sum_{\substack{i=1 \\
i \neq j}}^{n} \omega_{i} \\
= & 1 / 2 .
\end{aligned}
$$

ii) We have

$$
\begin{aligned}
& \operatorname{Pr}\left[\sum_{i=1}^{n} \omega_{i} x_{i} \leq x_{j}\right] \\
= & \frac{1}{2} \operatorname{Pr}\left[\sum_{i=1}^{n} \omega_{i} x_{i} \leq x_{j} \mid x_{j}=1\right]+\frac{1}{2} \operatorname{Pr}\left[\sum_{i=1}^{n} \omega_{i} x_{i} \leq x_{j} \mid x_{j}=0\right] \\
= & \left.\frac{1}{2}+\frac{1}{2} \operatorname{Pr}\left[\omega_{i} \neq 0 \Rightarrow x_{i}=0 \forall i \in N \backslash j\right)\right]=\frac{1}{2}+\frac{1}{2} \prod_{\substack{i \in N \backslash j \\
i \in j \\
\omega_{i} \neq 0}} \frac{1}{2} \\
= & \frac{1}{2}+\left(\frac{1}{2}\right)^{1+r}
\end{aligned}
$$


where $r=\left|\left\{i \in N \backslash j: \omega_{i} \neq 0\right\}\right|$.

\section{B Proof of Theorem 4.1}

(Sufficiency) Easy.

(Necessity) Consider first the case of veto indices. Let $\psi\left(\mathcal{C}_{v}, j\right)\left(j \in N, v \in \mathcal{F}_{N}\right)$ be numbers fulfilling the assumptions of the statement. By linearity and since the conversion formulas between $v$ and $a$ are linear (see Eqs. (2) and (3)), there exists $n 2^{n}$ coefficients $q_{T}^{j}(j \in$ $N, T \subseteq N)$ such that

$$
\psi\left(\mathcal{C}_{v}, j\right)=\sum_{T \subseteq N} q_{T}^{j} a(T) \quad\left(j \in N, v \in \mathcal{F}_{N}\right)
$$

Therefore, for any $T \subseteq N$, with $T \neq \varnothing$, and any $j \in N$, we have $\psi\left(\min _{T}, j\right)=q_{T}^{j}$ and hence

$$
\psi\left(\mathcal{C}_{v}, j\right)=\sum_{\substack{T \subseteq N \\ T \neq \varnothing}} a(T) \psi\left(\min _{T}, j\right) \quad\left(j \in N, v \in \mathcal{F}_{N}\right) .
$$

Now, for any $T \subset N$, with $T \neq \varnothing$ and $T \neq N$, and any $\pi \in \Pi_{N}$, we can easily show that

$$
\underline{v}_{\pi(T)}=\pi\left(\underline{v}_{T}\right) .
$$

Hence, by the symmetry axiom, we have

$$
\psi\left(\min _{T}, j\right)=\psi\left(\min _{\pi(T)}, \pi(j)\right) \quad(j \in N \backslash T)
$$

and we can set

$$
\psi_{t}:=\psi\left(\min _{T}, j\right) \quad(j \in N \backslash T) .
$$

But then, by the boundary axiom, Eq. (25) becomes

$$
\psi\left(\mathcal{C}_{v}, j\right)=\sum_{T \ni j} a(T)+\sum_{T \not \supset j} a(T) \psi_{t} \quad\left(j \in N, v \in \mathcal{F}_{N}\right)
$$

where we can set $\psi_{0}=0$ since $a(\varnothing)=0$.

Now, let $k \in\{2, \ldots, n\}$ and denote by $v_{\mathrm{OS}_{k}}$ the fuzzy measure associated to $\mathrm{OS}_{k}$. Since

$$
v_{\mathrm{OS}_{k}}=\pi\left(v_{\mathrm{OS}_{k}}\right) \quad\left(\pi \in \Pi_{N}\right),
$$

it follows, by the symmetry axiom, that

$$
\psi\left(\mathrm{OS}_{k}, i\right)=\psi\left(\mathrm{OS}_{k}, j\right) \quad(i, j \in N) .
$$

Therefore, combining the normalization axiom with Eq. (26), we obtain

$$
\begin{aligned}
n \frac{n-k}{n-1} & =n \operatorname{andness}\left(\mathrm{OS}_{k}\right)=n \psi\left(\mathrm{OS}_{k}, j\right)=\sum_{j \in N} \psi\left(\mathrm{OS}_{k}, j\right) \\
& =\sum_{j \in N}\left[\sum_{T \ni j} a(T)+\sum_{T \ngtr j} a(T) \psi_{t}\right]
\end{aligned}
$$


that is,

$$
n \frac{n-k}{n-1}=\sum_{T \subseteq N} a(T)\left[t+(n-t) \psi_{t}\right]
$$

where $a$ is the Möbius representation (8) of $v_{\mathrm{OS}_{k}}$.

Considering this latter identity for $k=2, \ldots, n$, we obtain the following triangular linear system

$$
\begin{aligned}
& \sum_{t=n-k+1}^{n-1}\left(\begin{array}{l}
n \\
t
\end{array}\right)(-1)^{t-n+k-1}\left(\begin{array}{c}
t-1 \\
n-k
\end{array}\right)(n-t) \psi_{t} \\
= & n \frac{n-k}{n-1}-\sum_{t=n-k+1}^{n}\left(\begin{array}{l}
n \\
t
\end{array}\right)(-1)^{t-n+k-1}\left(\begin{array}{c}
t-1 \\
n-k
\end{array}\right) t \quad(k=2, \ldots, n),
\end{aligned}
$$

that is,

$$
k\left(\begin{array}{l}
n \\
k
\end{array}\right) \sum_{t=n-k+1}^{n-1}(-1)^{t-n+k-1}\left(\begin{array}{l}
k-1 \\
n-t
\end{array}\right) \frac{n-t}{t} \psi_{t}=n \frac{n-k}{n-1} \quad(k=2, \ldots, n) .
$$

The unique solution of this system is given by

$$
\psi_{t}=\frac{n t-t-1}{(n-1)(t+1)} \quad(t=1, \ldots, n-1) .
$$

Indeed, this solution solves Eq. (27) since

$$
\begin{aligned}
\sum_{T \subseteq N} a(T)\left[t+(n-t) \psi_{t}\right] & =\sum_{T \subseteq N} a(T) \frac{n(n t-1)}{(n-1)(t+1)} \\
& =n \frac{1}{n-1} \sum_{T \subseteq N} a(T) \frac{n t-1}{t+1} \\
& =n \operatorname{andness}\left(\mathrm{OS}_{k}\right) \quad(\mathrm{cf} .(19)) \\
& =n \frac{n-k}{n-1} .
\end{aligned}
$$

The proof for favor indices is almost similar. Let $\psi\left(\mathcal{C}_{v}, j\right)\left(j \in N, v \in \mathcal{F}_{N}\right)$ be numbers fulfilling the assumptions of the statement. By linearity and since the conversion formulas between $v$ and $v^{*}$ and hence between $v$ and $a^{*}$ are linear, there exists $n 2^{n}$ coefficients $q_{T}^{j}$ $(j \in N, T \subseteq N)$ such that

$$
\psi\left(\mathcal{C}_{v}, j\right)=\sum_{T \subseteq N} q_{T}^{j} a^{*}(T) \quad\left(j \in N, v \in \mathcal{F}_{N}\right) .
$$

Therefore, for any $T \subseteq N$, with $T \neq \varnothing$, and any $j \in N$, we have $\psi\left(\max _{T}, j\right)=q_{T}^{j}$ and hence

$$
\psi\left(\mathcal{C}_{v}, j\right)=\sum_{\substack{T \subseteq N \\ T \neq \varnothing}} a^{*}(T) \psi\left(\max _{T}, j\right) \quad\left(j \in N, v \in \mathcal{F}_{N}\right) .
$$

Now, as for the veto indices, Eq. (29) becomes

$$
\psi\left(\mathcal{C}_{v}, j\right)=\sum_{T \ni j} a^{*}(T)+\sum_{T \ngtr j} a^{*}(T) \psi_{t} \quad\left(j \in N, v \in \mathcal{F}_{N}\right)
$$


where we can set $\psi_{0}=0$ since $a^{*}(\varnothing)=0$.

Now, let $k \in\{1, \ldots, n-1\}$. Similarly to the previous case, we have

$$
\begin{aligned}
n \frac{k-1}{n-1} & =n \operatorname{orness}\left(\mathrm{OS}_{k}\right)=n \psi\left(\mathrm{OS}_{k}, j\right)=\sum_{j \in N} \psi\left(\mathrm{OS}_{k}, j\right) \\
& =\sum_{j \in N}\left[\sum_{T \ni j} a^{*}(T)+\sum_{T \ngtr j} a^{*}(T) \psi_{t}\right]
\end{aligned}
$$

that is,

$$
n \frac{k-1}{n-1}=\sum_{T \subseteq N} a^{*}(T)\left[t+(n-t) \psi_{t}\right]
$$

where $a^{*}$ is the Möbius representation (10) of $v_{\mathrm{OS}_{k}}^{*}$.

Considering this latter identity for $k=1, \ldots, n-1$, we actually obtain the linear system (28) in which $k$ is replaced with $n-k+1$, and we have seen that the unique solution is given by

$$
\psi_{t}=\frac{n t-t-1}{(n-1)(t+1)} \quad(t=1, \ldots, n-1) .
$$

\section{References}

[1] T. Arnould and A. Ralescu, From "and" to "or", Lecture Notes in Comput. Sci., No. 682, Berlin, 1993, 119-128.

[2] A. Chateauneuf and J.Y. Jaffray, Some characterizations of lower probabilities and other monotone capacities through the use of Möbius inversion, Mathematical Social Sciences 17 (1989) 263-283.

[3] G. Choquet, Theory of capacities, Annales de l'Institut Fourier 5 (1953) 131-295.

[4] D. Dubois and J.-L. Koning, Social choice axioms for fuzzy set aggregation, Fuzzy Sets and Systems 43 (1991) 257-274.

[5] J.J. Dujmovic, Weighted conjunctive and disjunctive means and their application in system evaluation, Univ. Beograd. Publ. Elektrotechn. Fak. (1974) 147-158.

[6] J.J. Dujmovic, Extended continuous logic and the theory of complex criteria, Univ. Beograd. Publ. Elektrotechn. Fak. (1975) 197-216.

[7] J.M. Fernández Salido and S. Murakami, Extending Yager's orness concept for the OWA aggregators to other mean operators, Fuzzy Sets and Systems, in press.

[8] M. Grabisch, On equivalence classes of fuzzy connectives: the case of fuzzy integrals, IEEE Trans. Fuzzy Systems 3 (1) (1995) 96-109.

[9] M. Grabisch, The application of fuzzy integrals in multicriteria decision making, European Journal of Operational Research 89 (1996) 445-456.

[10] M. Grabisch, Alternative representations of discrete fuzzy measures for decision making, Int. J. of Uncertainty, Fuzziness, and Knowledge Based Systems 5 (1997) 587-607. 
[11] M. Grabisch, Alternative representations of OWA operators, in: R.R. Yager and J. Kacprzyk (eds.), The Ordered Weighted Averaging Operators: Theory and Applications, (Kluwer Academic Publisher, 1997) 73-85.

[12] M. Grabisch, The interaction and Möbius representations of fuzzy measures on finite spaces, $k$-additive measures: a survey, in: M. Grabisch, T. Murofushi, and M. Sugeno (eds.), Fuzzy Measures and Integrals - Theory and Applications. Studies in Fuzziness and Soft Computing Vol. 40 (Physica Verlag, Heidelberg, 2000) 70-93.

[13] M. Grabisch, J.-L. Marichal, and M. Roubens, Equivalent representations of set functions, Mathematics of Operations Research 25 (2000) 157-178.

[14] M. Grabisch, T. Murofushi, and M. Sugeno, Fuzzy measure of fuzzy events defined by fuzzy integrals, Fuzzy Sets and Systems 50 (1992) 293-313.

[15] U. Höhle, Integration with respect to fuzzy measures, Proc. IFAC Symposium on Theory and Applications of Digital Control, New Delhi, January 1982, 35-37.

[16] E.P. Klement, R. Mesiar, and E. Pap, Measure-based aggregation operators, Fuzzy Sets and Systems, to appear.

[17] A. Kolesárová, Parametric evaluation of aggregation operators, Proceedings of Mechanical Engineering, Bratislava, Slovakia, November 2001, pp. 684-689.

[18] J.-L. Marichal, Aggregation operators for multicriteria decision aid, Ph.D. thesis, Institute of Mathematics, University of Liège, Liège, Belgium, 1998.

[19] J.-L. Marichal, Behavioral analysis of aggregation in multicriteria decision aid, in: J. Fodor, B. De Baets, and P. Perny (eds.), Preferences and Decisions under Incomplete Knowledge. Studies in Fuzziness and Soft Computing Vol. 51 (Physica Verlag, Heidelberg, 2000) 153-178.

[20] J.-L. Marichal, An axiomatic approach of the discrete Choquet integral as a tool to aggregate interacting criteria, IEEE Transactions on Fuzzy Systems 8 (6) (2000) 800807.

[21] J.-L. Marichal, Aggregation of interacting criteria by means of the discrete Choquet integral, in: T. Calvo, G. Mayor, and R. Mesiar (eds.), Aggregation operators: new trends and applications. Studies in Fuzziness and Soft Computing Vol. 97 (PhysicaVerlag, Heidelberg, 2002) 224-244.

[22] J.-L. Marichal and M. Roubens, Determination of weights of interacting criteria from a reference set, European Journal of Operational Research 124 (3) (2000) 641-650.

[23] J.-L. Marichal and M. Roubens, On a sorting procedure in the presence of qualitative interacting points of view, in: J. Chojcan, J. Leski (eds.), Fuzzy sets and their applications (Silesian University Press, Gliwice, 2001) pp. 217-230 [ISBN 83-88000-64-0]. Also in: 22nd Linz Seminar on Fuzzy Set Theory (LINZ 2001), Linz, Austria, February 6-10, 2001, pp. 64-75. 
[24] T. Murofushi and M. Sugeno, An interpretation of fuzzy measure and the Choquet integral as an integral with respect to a fuzzy measure, Fuzzy Sets and Systems 29 (1989) 201-227.

[25] T. Murofushi and M. Sugeno, A theory of fuzzy measures. Representation, the Choquet integral and null sets, Journal of Mathematical Analysis and Applications 159 (2) (1991) 532-549.

[26] G.C. Rota, On the foundations of combinatorial theory I. Theory of Möbius functions, Zeitschrift für Wahrscheinlichkeitstheorie und Verwandte Gebiete 2 (1964) 340-368.

[27] L.S. Shapley, A value for $n$-person games, in: H.W. Kuhn and A.W. Tucker (eds.), Contributions to the Theory of Games, Vol. II, Annals of Mathematics Studies, 28, (Princeton University Press, Princeton, NJ, 1953) 307-317.

[28] M. Sugeno, Theory of fuzzy integrals and its applications, Ph.D. Thesis, Tokyo Institute of Technology, Tokyo, 1974.

[29] R.R. Yager, On ordered weighted averaging aggregation operators in multicriteria decision making, IEEE Trans. on Systems, Man and Cybernetics 18 (1988) 183-190.

[30] R.R. Yager, Quantifier guided aggregation using OWA operators, Int. J. Intelligent Systems 11 (1996) 49-73. 